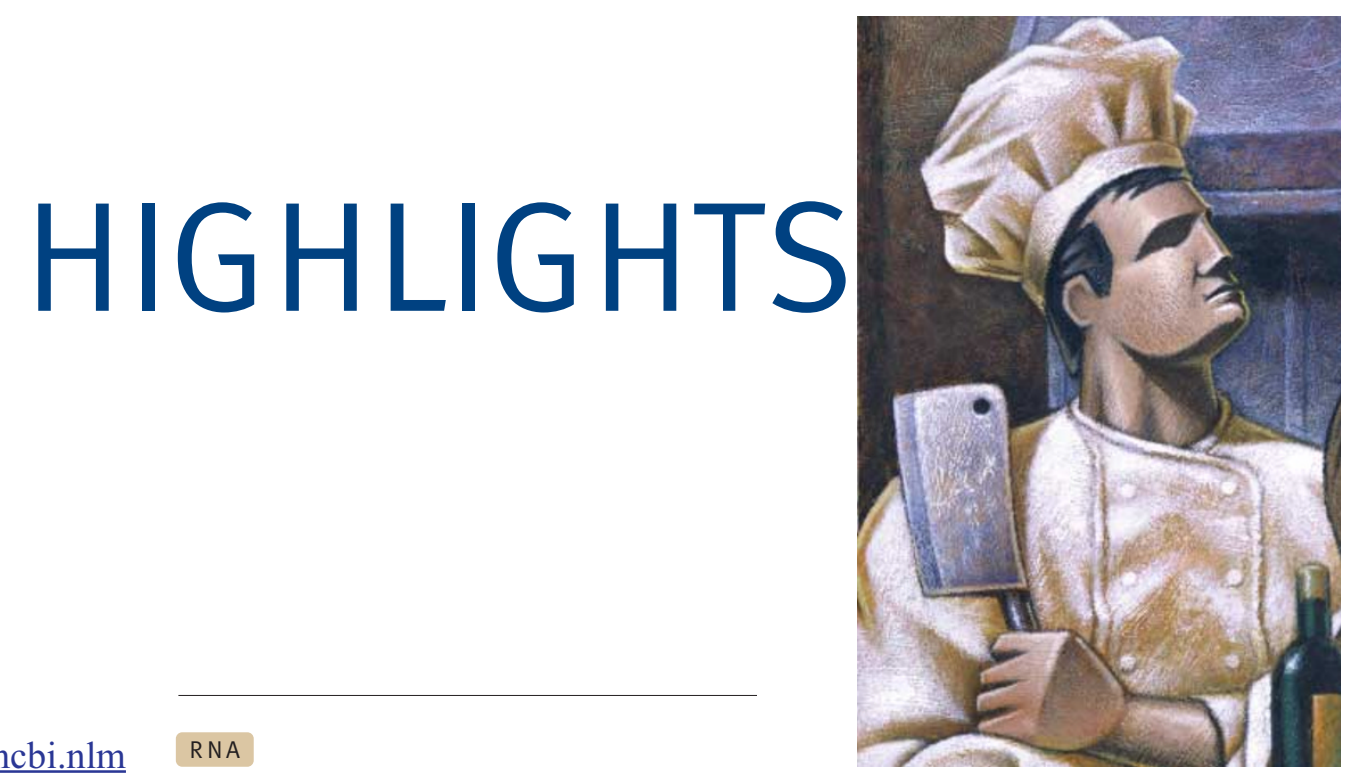

let-7

http://www.ncbi.nlm .nih.gov/locuslink/L ocRpt.cgi? $=266952$

Dicer

http://us.expasy.org/

cgi-

bin/niceprot.pl?Q9U $\underline{\text { PY3 }}$

\section{Chop, chop}

Gene silencing by RNA interference (RNAi) is mediated by small RNAs, such as microRNAs (miRNAs), which guide the selection of silencing targets. The selected messenger RNA can be blocked to prevent translation, or is cleaved and degraded by an enzyme complex known as RISC (RNA-induced silencing complex). Reporting in Nature, the groups of Hannon and Plasterk reveal a new component of RISC, which contributes to RNA degradation. And, in a second report, Kim and colleagues identify a new miRNA-processing enzyme.

Hannon, Plasterk and colleagues purified the Drosophila melanogaster RISC complex and identified a new factor. This new protein contains five repeats of a staphylococcal/micrococcal-nuclease domain as well as a tudor domain and was therefore called Tudor-SN (tudor staphylococcal nuclease). Tudor-SN was also found in Caenorhabditis elegans and mammalian RISC complexes.

When the authors expressed recombinant Drosophila Tudor-SN, a nuclease activity co-fractionated with the recombinant protein, and this activity was reduced by adding Tudor-SN anti-peptide antibodies. Using a lacZ-reporter system in $C$. elegans that was under the translational control of let7 miRNA, Hannon, Plasterk and co-workers showed that the RNAi-induced depletion of Tudor-SN resulted in the continuing expression of the reporter gene. So, Tudor-SN in C. elegans is necessary for let7-mediated gene silencing in vivo.

A specific inhibitor of staphylococcal nucleases blocked Tudor-SN activity and, importantly, also RISC activity. Nevertheless, the authors hesitate to conclude that Tudor-SN is the sole nuclease responsible for RNAi. This is because, in contrast to RISC, recombinant Tudor-SN does not have sequence specificity, and it cleaves both RNA and DNA (no DNase activity has been detected in RISC). Also, its activity cannot explain the specific mRNA cleavage pattern that has been reported for RISC.

So, Hannon, Plasterk and colleagues propose that RISC contains multiple nucleases, one of which catalyses site-specific mRNA cleavage, and that Tudor-SN might degrade the remainder of the mRNA. Future studies are expected to reveal additional RISC-associated nucleases.

In the second paper, Kim and colleagues investigated miRNA biogenesis, which is a two-step process. First, primary miRNA transcripts (primiRNAs) are processed in the nucleus to produce $\sim 70$-nucleotide stem-loop pre-miRNAs. Next, a second, cytoplasmic processing step mediated by Dicer — an RNase III enzyme - results in the production of mature miRNA.

In their quest to identify the enzyme responsible for the first processing step, Kim and co-workers mapped the sites of cleavage in the $5^{\prime}$ and $3^{\prime}$ ends of pre-miRNA. The stem loop had a two-nucleotide overhang at its $3^{\prime}$ end, which is characteristic of an RNase-III-mediated cleavage reaction. The double-stranded (ds)RNA stem structure is essential for efficient processing, which indicates the involvement of a dsRNA-specific endonuclease such as Drosha - an RNase III enzyme that is present in the nucleus.

To explore this possibility, the authors expressed tagged Drosha in cells and isolated the protein by immunoprecipitation. The precipitate was then used in an in vitro RNAprocessing reaction, which yielded 60-70-nucleotide fragments that corresponded to pre-miRNAs.

Kim and colleagues then carried out RNAi to deplete Drosha in cultured cells, which resulted in the accumulation of pri-miRNA and a decrease in pre-miRNA levels in vivo. Next, they tested a range of other miRNAs, which gave similar results, so the authors concluded that Drosha is probably widely used for miRNA processing.

Now that the two enzymes responsible for miRNA biogenesis Drosha and Dicer - are known, it will be important to understand how these enzymes are regulated.

Arianne Heinrichs

(2) References and links

ORIGINAL RESEARCH PAPERS Caudy, A. A. et al. A micrococcal nuclease homolog in RNAi effector complexes. Nature 425, 411-414 (2003) | Lee, Y. et al. The nuclear RNase III Drosha initiates microRNA processing. Nature $\mathbf{4 2 5}, 415-419$ (2003)

FURTHER READING Liu, A. et al. R2D2, a bridge between the initiation and effector steps of the Drosophila RNAi pathway. Science $\mathbf{3 0 1}$, 1921-1925 (2003) 